\title{
ESGCT 2015 Abstract Author Index
}

Aagaard, L, P186, P257

Aalbers, C J, P220

Aatonen, M, OR058

Abad, J L, P039

Abdul-Razak, H H, P121

Abdullah, S, OR040

Abed, S, P207

Abel, T, OR033

Ablinger, M, P058

Ackermann, M, P200

Adachi, T, P079

Adjali, O, OR011

Aerts-Kaya, F, P212, P213, P214

Afzal, S, OR022, P083, P237

Agaugue, S, OR025, P087, P109

Agudo, J, P134

Aguiar, T, P156

Ahonen, M, P015

Aid-Launais, R, OR066

Aiolfi, R, OR035

Airaksinen, A J, P018

Airenne, K J, P049

Aiuti, A, OR056, OR065, P081, P104, P242, P269

Akahori, Y, P091

Akar-Soycan, I, P076, P077

Akbaba, H, P064, P171

Alberquilla, O, P007

Albertini, P, P242

Alemany, R, P022, P089, P106

Alexander, I E, OR075, P098, P099, P265

Alexander, S I, P098

Alfano, A L, P023

Alfranca, A, P194

Aliño, S F, P034, P169

Ali, R R, OR021, P187

Alici, E, OR013

Alievi, L, P029

Alitalo, K, P030

Alliegro, M, P133

Almarza, E, OR050

Alonso-Ferrero, M E, P121

Alonso, D Balboa, P206

Alton, E W, OR049

Altunbas, H A, P137, P241

Álvarez, V, P039

Alvarez, L, P054

Alves, L S, P190

Alves, P M, OR071, P145, P156, P191, P244, P247

Alves, P. M., P273

Amaishi, Y, P091
Ameline, B, P181

Amiot, B, OR046

Amor, F, P158

Amrolia, P, OR037

Anastasiadis, K, OR001

Andaloussi, S EL, P170

Andaluz, A, P128

Andersen, C B F, P044

Andolfi, G, P086

André-Schmutz, I, OR037

Andriolo, G, OR060

Angus, P W, P265

Añor, S, P128

Anselmo, A, OR035

Antoniou, M N, P003

Antonitsis, P, OR001

Antopolsky, M, OR052, P088

Anttila, M, P030

Antunes, A S L, P122

Aranyi, T, OR027

Arbuthnot, P, P256

Arendt, A, P028

Ariga, T, P094

Armentano, D, P008

Armstrong, D K, OR049

Arnaudova, R, P028, P029

Arntz, O J, P110

Arribas, B, P208

Arroyo, M, P194

Artaud, C, OR031

Askou, A L, P186, P257

Asokan, A, OR006

Athanasopoulos, T, OR038

Athanassiadou, A, P177

Audrain, M, OR033

Auricchio, A, P133, P250

Ausseil, J, OR031

Auvinen, T, OR067

Aviv, V, P138

Ayala-Breton, C, OR054

Aydin, G, P212, P213

Ayuso, E, P141

Azam, S A, OR021

Babarit, C, P157

Bacchetta, R, OR030, OR048

Bacci, M L, P250

Badin, R A, P188

Baekelandt, V, P264

Bagel, J, P124

Bak, R O, OR030

Baker, A H, P234

Balboa, D, OR015
Balci, M K, P228

Ballmaier, M, P226

Banfi, A, OR064

Baranov, V S, P065, P176

Barbas III, C F, P063

Barile, L, OR060

Barrio, L C, P209

Bartholomä, C, OR022

Bartholoma, C, P083

Bartholomae, C C, P121, P239

Bartolaccini, S, P227, P261

Basilico, C, OR010

Basu, U, OR006

Bauche, C, OR025, P087, P109, P142, P143

Bauer, J W, P058, P071, P072, P073

Bauer, R, OR009

Baugnon, T, OR031

Baum, C, OR024

Beck, K, P021

Becker, F, P085

Bedel, A, P217

Beekman, J, P264

Beggs, A H, P158

Behr, M, P112

Bejanariu, A, OR025, P109, P142

Bek, T, P186

Beley, C, P183, P251

Beliveau, F, P217

Bell, P, P124, P253

Bello, A Buj, OR043, P158

Belt, H, P101

Bemelmans, A P, OR033

Benabdellah, K, P082, P230

Benchaouir, R, P183, P251

Benedetti, S, OR042

Benedicenti, F, P125

Benedicenti, F., P274

Bennink, M B, P110

Bermúdez, I R, P023

Bernad, A, OR071

Bernardo, M E, P086

Berneman, Z, OR068, P219

Berta, G N, P005

Berthelette, P, P008

Bertolino, P, OR075, P099

Bertolino, P J, P098

Bertoni, A, P021

Bessodes, M, OR051

Bevaart, L, P220

Bevilacqua, M, P225

Beyer, S, OR073

Bezzina, C R, P040 
Biagi, D G, P205

Biasco, L, OR056, OR065, P269

Biffi, A, P125, P129, P132, P242

Biget, M, P181

Bigger, B, OR044, P122

Bijvelds, M, P264

Binder-Scholl, G, P266

Binley, K, P188

Bishop, G A, OR075, P098, P099

Bitoque, D B, P174, P190

Blackford, S J, P187

Blanca, V, P194

Blankenstein, T, P100

Blinova, M, P197

Blinova, M I, P195, P199, P203

Blits, B, OR029, P060

Blomberg, P, P170

Blouin, V, P141

Blum, N M, P199

Blum, S, OR036

Blundell, M, P121

Bobis-Wozowicz, S, P074, P215

Bogacheva, M S, P065, P176

Boink, G JJ, P040

Bolis, S, OR060

Bondanza, A, OR003, OR065, P086, P090, P095

Bonini, C, OR003, OR065, P086, P090, P095

Bonnamy, B, P246

Bonnin, D, P146

Booth, C, P117

Bordignon, C, OR003, OR065, P090, P095, P144

Borlado, L R, OR071

Borquez-Ojeda, O, P012

Boruczkowski, D, P074, P215

Bosch, F, P128, P131, P134

Bossi, S, P144

Bosticardo, M, OR035, P092

Boudeffa, D, P149

Bouquet, C, OR020

Bourg, N, P160

Boussicault, F, P148

Bouzelha, M, P141

Bovolenta, C, OR012, P144

Bovolenta, M, OR076

Bovy, T, P138

Bowen, D G, OR075, P098, P099

Boyd, A C, OR049

Boyer, O, P118

Bozzola, V, P029

Bradshaw, A, P234

Bragonzi, A, P081

Bramante, S, P236

Brasca, S, P125

Braudeau, J, OR033

Braulke, T, P127

Bredius, R G, P108

Breitenbach, J S, P058

Brendel, C, P116

Brennig, S, P200

Bressin, R K, OR006

Brito, C, OR071, P191
Brkic, S, OR064

Broekstra, N, OR057, P220

Broeren, M GA, P110

Broissand, C, OR031

Broucque, F, P141

Bruckner-Tudermann, L, P073

Brugman, M H, P108

Bruhn, L, OR030

Buchholz, C F, OR033

Buchholz, C J, OR054, OR073

Buckland, K, P111

Buclez, P, P251

Buening, H, P152

Bueren, J A, OR034, OR050, P001, P002, P007, P053, P054, P055, P121, $\mathrm{P} 210$

Bulfone, A, OR032

Bumcrot, D, P075

Bunker, A, OR052, P088, P103

Bunker, D LJ, P099

Bunning, H, P070

Burnham, B, P008

Buscail, L, OR002

Buscara, L, P158

Cabrera, G, P130, P232

Cafferata, E A, OR004

Cagnan, I, P076, P077, P204

Calabria, A, OR023, P125, P262

Calabria, A., P274

Calado, S M, P167, P175, P190

Calatayud, C, OR014

Calcedo, R, P253

Calero-Garcia, M, P062

Calise, D, P048

Calne, R Y, P135

Camadro, J M, P164, P231

Camisa, B, OR003, P086, P090

Canatan, H, P241

Candia, A N, P023

Canovas, S, P208

Cantore, A, P004, P006, P227

Capasso, C, OR052, P015, P088, P103, P225

Capo, V, OR019, P081, P092

Carlon, M S, P264

Carmo, M, P062

Carrascon, V C, P103

Carrera, P, P092

Carriglio, N, P104, P242

Carrondo, M J T, OR071, P145, P150, P156, P191, P247

Cartier, N, OR033

Casado, J A, OR034

Casal, M L, P124

Casana, E, P131, P134

Casellas, A, P134

Casey, N P, P011

Castiello, M C, OR019, OR035, P092

Casucci, M, OR003, P090

Cataliotti, A, OR047

Catamancio, S La Seta, P028, P029

Cathomen, T, OR014, P003, P053, $\mathrm{P} 074, \mathrm{P} 226$
Cattoglio, C, P061

Catzín-Kuhlmann, A, P130

Catzin-Kuhlmann, A, P232

Cavalier, M, P080

Cavazzana, M, OR037

Cavazzin, C, OR032

Çetintas, V B, P178

Cecere, F, P132

Cejalvo, T, P022

Cereso, N, P085

Cerullo, V, OR052, P015, P027, P088, P103, P225

Cervio, E, OR060

Cesana, D, OR023, P262

Cesana, D., P274

Cevher, I, P214

Chalberg, T W, P147, P189, P249

Chamorro, C, P070

Chan, J, P147

Chandler, R J, P126

Chang, K H, OR017

Chao, J, P240

Chao, L, P240

Charbonnier, S, P258

Charrier, M, OR011

Charrier, S, P061, P111

Charton, K, P159, P160, P161

Chaufour, X, P048

Chen, H, P147

Chen, K, OR017, P004

Chen, S Y, OR027

Chen, X, OR041

Cheng, S H, OR049

Chernin, G, OR036

Chester, J D, P224

Cheung, G WC, OR072

Chiang, C, OR013

Childers, M K, P158

Chinchon, R, P053

Chiriaco, M, P081

Choa, R, P124

Choi, U, OR045

Christoffels, V M, P040

Chrobok, M, OR013

Chuah, M, P006

Chuah, M K, OR038

Chudasama, P, P057

Chumakov, P M, P254

Cibelli, J B, P208

Cicalese, M P, OR056

Ciceri, F, OR003, OR065, P086, P090, P095

Cicero, V Lo, OR060

Cieri, N, OR065

Ciré, S, P096, P114

Cittaro, D, P261, P269

Ciudad, C J, P034

Clark, J T, OR030

Clark, K R, P154

Clarkson, N G, OR078

Claudiani, P, P133

Cobo, M, P082, P230

Cocchiarella, F, P129

Cohn, R D, OR040 
Colangelo, D, P005

Colella, P, P250

Collaud, F, OR069

Collins, M A, P075

Collinson, L M, P191

Colombi, S, P095

Combal, J P, OR020

Comi, M, OR032

Conscience, A, P080

Consiglio, A, OR014

Conway, A, OR012

Cordelier, P, OR002

Corna, S, P144

Cornils, K, P127

Coroadinha, A S, P145, P244, P247

Coroadinha, A. S., P273

Corre, G, P061

Corydon, T, P186

Corydon, T J, P257

Cossu, G, OR042

Cost, G J, OR012

Costa, A R, P174

Cota, M, P028, P029

Cote, C, P140

Coughlan, L, P224

Crack, L, P016

Cristofori, P, P104, P132, P242

Cuccovillo, I, P261

Cuneo, N, P023

Cunha, B, P156

Cunningham, E C, OR075, P098, P099

Cunningham, S, OR049, OR075, P098

Cunningham, S C, P265

Curiel, D T, OR004

D'Agostino, M, OR065

D'Angelo, A, P006

da Cunha, M F, P264

Daans, J, OR068, P219

Dabernat, S, P217

Dagnaes-Hansen, F, P257

Dam, N, P039

Damodar, K, P080

Danièle, N, OR043

Danos, O, OR017, P004

Dariolli, R, P205

David, A L, P275

David, R, P168

Davies, G, OR049

Davies, J A, P224

Davies, J C, OR049

De Bleser, P, OR038

De Boeck, K, P264

De Bournonville, S, OR031

De Cicco, S, OR032

de Heredia, C Diaz, OR034

de Jonge, H, P264

De Luca, V, P085

de Saint Basile, G, OR037

de Simone, S, P250

de Sio, F R Santoni, OR023, OR048

de Verneuil, H, P217

De Vocht, N, OR068, P219

De Vos, J, P085 de Vrij, J, OR057

DeRavin, S S, OR045

Debyser, Z, P263, P264

Deglon, N, OR029

Deichmann, A, OR022

Deiva, K, OR031

Dekkers, J D, P264

del Castillo, N, P022

del Portillo, I, P022

Del Rio, M, P070

Delacroix, N, P142

Delai, S, P132, P242

Delalande, A, OR051

Dellinger, D, OR030

Demeulemeester, J, P263

Demidova, E V, P254

Denard, J, P158, P231

Denby, L, P234

Deplaine, G, P142, P143

Deroyer, J, P142, P143

Deschamps, J Y, P181

Deventer, S V, OR029

Dhainaut, M, P219

Di Matteo, G, P081

Di Serio, C, P269

Di Stefano, A, OR066

Di Tomaso, T, OR012, P227

Diaconu, I, P236

Diarra, S, P066

Dias-Florencio, G, P251

Dickson, G, OR038

Dickx, Y, P146

Dieringer, B, P271

Dietrich, D, P028

Diez, B, P054

Dijkstra, M H, P044, P050

Dionisio, F, OR056, OR065, P269

Dobrodumov, A, P102

Doering, J, P158

Donnou-Triffault, S, P024, P114

Dooley, D, OR068, P219

Dowling, J J, OR040

Drager, D, P004

Draghici, E, OR035, P092

Drugmand, J C, P138

Du, F, P012

Duarte, B, P070

Dubart-Kupperschmitt, A, P207

Dubreil, L, P157

Dubuisson, L, P217

Dudek, N, P098

Dugovic, B, P183

Dulak, J, P113

Duran, Y, OR021, P187

Duru, A D, OR013

Edelman, A, P264

Efrati, S, OR036

Egorova, A A, P065, P176

Ehrhardt, A, P112

Eksi, Y E, P228

El-Khatib, M, P196

Eleftheriadou, I, OR039

Elgilani, F, OR046
Elgmati, H, OR049

Ellis, S, OR078

Ellison, S M, OR044

Ely, A, P256

Emmerling, V V, OR007

Enns, H, OR009

Erel, G, P171

Erman, B, OR013

Ertan, G, P178

Escobar, G, OR012, OR048

Esendagli, G, P076, P077

Esparza, A, P130, P232

Estévez, F J Molina, P121

Eurola, S, OR015, P206

Evans, M J, OR001, P198, P211

Even-Desrumeaux, K, P087

Ezzine, S, OR051

Fañanas-Baquero, S, P002

Faella, A, OR014

Falcone, L, OR003, P090

Fang, J W, P075

Farina, I G, P238

Farinelli, G, P081

Farley, D C, OR078

Faulkner, A, OR061

Fechner, H, P271, P272

Fehse, B, P013, P127

Fenard, D, P149

Ferber, S, P138

Ferla, R, P133

Fernández-García, M, P210

Fernández, I, OR014

Fernandes, P, P191

Fernandez-Muñoz, B, P208

Ferrari, G, OR042, P242

Ferro, F, P132, P242

Ferrua, F, OR056

Fibbe, W E, P108

Fillat, C, P221

Finckenberg, P, OR058

Finn, J D, OR057, P220

Finocchi, A, P081

Firrito, C, OR012

Fischer, S, OR007

Fisson, S, P024, P114

Fitoussy, S, OR020

Flaschel, E, P139

Fol, R, OR033

Follenzi, A, P005

Fontana, E, P092

Fontanellas, A, OR005

Formas-Oliveira, A S, P145, P247

Fortino, V, P015

Fröhling, S, P057

Fracchia, S, P028, P029

François, A, P141

Fransen, E, P219

Frati, G, OR032

Freiling, R T, OR073

Frenzel, E, P200

Friedel, T, OR054

Friedland, A E, P075

Friehs, K, P139 
Frizzale, G, P029

Fronza, R, OR022, OR065, P083, P237, P238, P239

$\mathrm{Fu}, \mathrm{Z}$ Y, P135

Fuchsluger, T, P152

Fujiwara, H, P011, P091

Fuster, J, P230

Gabriel, R, P093, P121, P237

Gaj, T, P063

Galetto, R, P002, P055

Galibert, L, P146, P252

Galibin, O, P197

Galli, E, P086

Gallina, P, OR023, P262

Galvani, G, P082

Galy, A, OR020, OR027, OR034, P024, P096, P114, P149

Gambera, S, P194

Gan, C H V, P121

Gan, S U, P135

Gancberg, D, P202

Gangemi, M A, P023

Garín, M I, P121

García-Castro, J, P194

García, M, P128

Garcia-Castro, J, P022

Garcia-Gomez, M, P007

Garcia, C, P164, P231

Garcia, L, P183, P251

Garcia, M, P131

Garmy-Susini, B, P048

Garofalo, M, OR052, P015, P088, P103, P225

Gasmi, M, P147, P249

Gaspar, B, P111

Gaspar, H B, P062, P117

Gayral, M, OR002

Gebretsadik, K, P147

Geis, F, P260

Genovese, P, OR003, OR012, OR019, P054, P090

Gentner, B, OR012, P081, P129

Giannakopoulos, A, P177

Gianni-Barrera, R, OR064

Gicquel, E, P161

Gidekel, M, OR004

Gijsbers, R, P263, P264

Gil-Farina, I, OR005

Gill, D R, OR049

Giordano, F A, P239

Gissberg, O, P056

Giuliani, E, OR012, P144

Giunti, M, P250

Givissis, P, P198

Gläser, A, P078

Glass, C K, P042

Glimm, H, P057

Glorioso, J, OR046

Goddard, M, P158

Godinho, T, P226

Gojo, S, P079

Gomes-Alves, P, OR071

Gomes, C P, P166
Gomez-Lazaro, M, P166

Gonçalves, C, P180

Gonçalves, J, P063

Gonçalves, M AFV, OR041

Gonorazky, H, OR040

González-Muñoz, E, P208

Gonzalez-Aseguinolaza, G, OR005

Gonzalez-Cordero, A, P187

Goode, T, P253

Goossens, H, OR068, P219

Gori, J L, P075

Gouble, A, P002, P055

Gougeon, M L, OR031

Goumans, M JTH, P040

Gourlay, J, P188

Goyenvalle, A, P170, P183

Grönberg-Vähä-Koskela, S, P018

Granados, M, P232

Grange, R W, P158

Greco, D, P015

Greco, R, OR065, P095

Greening, A, OR049

Gregori, S, OR032, P086

Gregory, P, OR017, P054

Gregory, P D, OR012, OR019, OR045, P082, P121, P227

Grez, M, P081, P116, P260

Griesenbach, U, OR049

Griffith, G, P183

Grimm, D, OR022

Gritti, A, OR032

Groppa, E, OR064

Gruenert, A, P152

Güenechea, G, P121

Guenechea, G, OR034, OR050, P055

Guerreiro, M R, P145, P244

Guevel, L, P157

Guglielmetti, C, OR068

Guianvarc'h, L, OR069, P148

Guillou, L, P080

Gullotta, F, P086

Gunel-Ozcan, A, P214

Gurda, B L, P124

Gurzeler, E, P041, P044

Guschin, D, OR012

Guse, K, P015, P222

Gutierrez-Guerrero, A, P082, P230

Gutmann, T, P222

Guzhova, I, P102

Hämäläinen, K, P030

Hänseler, W, P116

Ha, T C, OR024

Haab, G Acosta, OR004

Hack, A, P012

Hadri, A, P142, P143

Hainzl, S, P058, P071, P072, P073

Hakkarainen, H, P030, P052

Hakulinen, M, OR067

Hakumäki, J, OR067

Halldén, G, OR053

Halonen, P, OR008

Hamel, A, OR011

Hamel, C P, P080, P085
Hamidi, S, OR066

Hammond, S, P170

Hanauer, J D S, OR054

Handrick, R, OR007

Hanoun, N, OR002

Hansen, G, OR055, P200

Hantelys, F, P048

Hantraye, P, P188

Happle, C, OR055, P200

Harman, K, OR049

Harmening, N, P182, P184

Hartikainen, J, OR008

Hartmann, J, OR073

Haskins, M E, P124

Hassin, S, P138

Hassinen, M M, OR070, P151, P153, P223, P245

Haurigot, V, P128

Havunen, R, P112, P236

Hayden, M, OR029

$\mathrm{He}, \mathrm{Q}, \mathrm{P} 012$

He, Z, P253

Heard, J M, OR031

Heath, J M, P075

Hebben, M, OR069, P111, P148

Heberer, M, OR064

Hedman, M, P046

Heikkilä, K M, OR070, P153

Heikura, T, OR063, OR070, P049, P155

Heinäniemi, M, P043

Hemminki, A, P015, P017, P018, P112, P222, P236

Hemminki, O, P236

Henckaerts, E, P122, P237

Hendel, A, OR030

Hendrix, S, OR068, P219

Hens, N, OR068

Herath, C B, P265

Herchenröder, O, P078

Hermann, K, P268

Hernandez, R J, P242

Hernandez, R Jofra, P081

Hernando, M, P210

Herrera, G, P210

Herrero, E Senís, OR022

Herrero, M J, P034, P169

Hervas, L Abelleira, OR021

Hetzel, M, OR055

Hickey, R D, OR046

Higgins, T, OR049

Hildebrandt, S, P078

Hinderer, C, P124

Hinkkanen, A, P270

Hiroshima, K, P014

Hirsch, M L, P160

Hirvinen, M, OR052, P015, P088, P103, P225

Hoerer, M, OR007

Hoffmann, D, P226, P260

Holappa, L, P276

Holditch, S J, OR047, OR074

Hollenhorst, M I, P264

Hollensen, A K, P186

Holmes, M, P054 
Holmes, M C, OR012, OR019, P082, P121

Holmgaard, A, P186

Holstein, M, OR050

Holthaus, S M kleine, OR021

Honda, T, OR074, P255

Hoornaert, C, OR068, P219

Hocquemiller, M, OR026

Horner, P J, P208

Horvat, L, OR051

Hoshiya, H, OR042

Hosomi, K, P188

Hossain, J A, P013

Houghton, B, P117

Hovnanian, A, OR076, P258

Howe, S J, P121

$\mathrm{Hu}, \mathrm{M}, \mathrm{P} 098$

Hukkanen, V, P105, P233

Hüttner, C, P071, P072

Huopio, H, OR015

Husso, T, OR028, P035, P036

Husson, B, OR031

Huusko, J, P041, P044, P046, P050, P276

Huusko, V, P151

Hyatt, E, OR040

Hyde, S C, OR049

Hytönen, E, P030, P276

Hytönen, J, OR067

Hytonen, J P, OR008

Iannacone, M, OR035

Igarashi, Y, P094

Ikeda, H, P091

Ikeda, Y, OR006, OR046, OR047, OR074, P123, P196

Ikegami, M, OR059

Imbert, M, P183

Immonen, A, P270

Impola, U, OR058

Incao, A A, P126

Innes, J A, OR049

Inverso, D, OR035

Iodice, C, P250

Iovine, B, P225

Iqbal, S, OR078

Irvine, E, OR039

Ishida, R, P079

Itaka, K, OR059, P173

Ivakine, E, OR040

Ivanova, A, P197

Ivics, Z, OR050, P066

Izmiryan, A, OR076

Izsvák, Z, OR050, P066, P182

Izsvak, Z, P184

Izumiya, Y, OR006

Jääskeläinen, J E, P270

Jääskelainen, M, P151

Jackson, R, P008

Jacobsen, M, P257

Jacobus, E J, P123, P196

Jaeschke, N M, P226

Jaffres, P A, OR051
Jagot, S, P157

Jakobsen, B, P266

Jambrina, C, P131, P134

James, N D, P016

Jamet, T, OR043

Jamiluddin, M, P109

Jan, C, P188

Janciauskiene, S, P200

Janssen, J M, OR041

Jaulin, N, OR011

Jazwa, A, P113

Jenal, U, OR062

Jenny, C, P231

Jensen, T G, P257

Jiang, H, OR017, P004, P006

Jiménez, S, OR014

Jimenez, C, P022

Jimenez, V, P131, P134

Johnen, S, P066, P182, P184

Johnston, I, OR072

Jones, P, P188

José, A, P221

Jose, A, P221

Joubert, R, OR043

Jouen, F, P118

Jozkowicz, A, P113

Judintceva, N M, P199

Julien, L, OR043

Jungmann, A, OR009

Kärjä, V, P046

Kaartinen, T, P101

Kaeppel, C, OR005

Kaikkonen, M U, OR063, P038, P042, P043, P049, P050, P252

Kaiser, A, OR072

Kaiser, R J, OR030

Kajaste-Rudnitski, A, P081, P261

Kalatzis, V, P080, P085

Kami, D, P079

Kammertoens, T, P100

Kamycka, E, P215

Kan, O, P266

Kanerva, A, P222

Kang, K S, P267

Kang, M, P026

Kankuri, E, OR058

Kantarci, A G, P064, P171, P178

Karam, N Laham, P229

Karhinen, M, OR070, P151, P153

Karioja-Kallio, A, P027

Karttunen, T, P193

Karvonen, A, P030

Kasahara, N, P268

Kataoka, K, OR059, P173

Katus, H A, OR009

Kawamura, K, P014

Kazuki, Y, OR042

Kelleher, M, P188

Kelton, W, OR016

Kemaladewi, D U, OR040

Kennedy, A B, OR030

Keppler, O T, P239

Keravala, A, P249
Keren, K Doenyas-Barak, OR036

Kern, W, P239

Keyaerts, M, OR038

Khebizi, Q, P114

Khodosevich, K, OR073

Kilpinen, L, OR058

Kilpinen, S, OR058

Kim, J, P026

Kim, T Y, P107, P267

Kim, Y -S, P026

Kinnon, C, P121

Kinnunen, K, P193

Kirchhof, J, P021

Kirita, Y, P079

Kiselev, A V, P065, P176

Kitzmüller, S, P073

Kitzmüller, S K, P058

Kivelä, A M, P044

Klapwijk, J, P104

Kleanthous, M, P003

Klerk, M, P040

Klimenkova, O, P200

Klopfleisch, R, P271, P272

Kmiotek, K, P074, P215

Knickrehm, J, P127

Kniffin, T, P147, P249

Knipping, F, P093

Kobelt, D, OR050

Koch, J, OR073

Koch, W J, OR009

Kochanek, S, OR007

Kocher, T, P058, P072, P073

Kochneva, G V, P254

Koide, K, OR006

Koistinen, A, OR008

Kokki, E, P193

Kolari, I L, OR028, P035, P036

Koller, U, P071, P072, P073

Kollmann, K, P127

Koltsova, A M, P216

Komorizono, R, OR074

Konstantinova, P, OR029, P060

Koontz, S, OR045

Kootstra, N, P239

Koponen, J, P101

Koponen, S, P270

Kopru, C Z, P076, P077

Korhonen, M, P101

Korkusuz, P, P076, P077

Korpisalo, P, OR067, P037

Korshunov, A, P021

Korte, M, OR033

Koskenvuo, J, P045

Kosma, V M, P030

Kotmakçi, M, P178

Kräusslich, H G, P239

Kramer, E, P220

Kraszewska, I, P113

Kremer, E J, P191

Krieger, J E, P205

Kropp, M, P182, P184

Kruczek, K, P187

Kubo, S, P014, P268

Kudva, Y C, P123, P196 
Kuehlcke, K, P258

Kuehle, J, P260

Kuhn, A, OR055

Kuivanen, A, OR008

Kujala, J, P193

Kume, A, P010

Kunduzova, O, P048

Kurmi, K, P123

Kurreck, J, P272

Kuryk, L, OR052, P015, P027, P088, P103, P225

Kusaba, T, P079

Kustikova, O, OR024

Kuzushima, K, P011

Kyostio-Moore, S, P008

Lázaro-Ibáñez, E, P019

Laakkonen, J, OR008

Laakkonen, J P, OR063, P038, P049, P052

Laborda, E, P022

Labruna, G, P047

Lachmann, N, OR055, P200

Lad, Y, OR078, P188

Laham-Karam, N, P046

Lahoutte, T, OR038

Laine, A, P041, P044

Laitinen, P, OR028, P035, P036

Laitinen, S, OR058

Laitinen, T, OR067

Lamana, M, P210

Lamana, M L, OR034

Lambiase, A, OR065, P095

Lambricht, L, P097

Lamrissi-Garcia, I, P217

Lange, L, P260

Langerak, A W, P108

Langford-Smith, A, OR044

Langford-Smith, K, OR044

Lankester, A C, P108

Lappalainen, J P, P038

Larcher, F, P070

Larcher, T, P157

Lardenois, A, P157

Laricchia-Robbio, L, P208

Latournerie, V, P158

Laufs, S, P239

Law, N, P266

Lawlor, M W, OR043, P158

Lazarevic, D, P261

Lazarus, G G, P165

Le Bec, C, OR069, P148, P158

Le Blon, D, OR068, P219

Le Meur, G, P181

Le Visage, C, OR066

León, X, P128

Lebas, B, P048

Leber, S, P123

Leborgne, C, P118

Leboulch, P, P207

Lecomte, E, P141

Lederer, C W, P003

Ledevin, M, P157

Lee, G, OR045
Lee, J, OR045

Lee, J H, P267

Lee, K O, P135

Lee, S W, P107

Lees, M, P275

Leger, T, P164, P231

Leggiero, E, P047

Lehtinen, J, P233

Leiro, V, P166

Leisegang, M, P100

Lemarié, A, OR002

Lemmens, E, OR068, P219

Lener, T, P073

Lengler, J, P227

Lennaertz, A, P029

Lenz, J., P274

Leon, X, P131, P134

Leonardelli, L, OR056, P269

Lerner, B, OR036

Leroux, I, OR011

Lesch, H P, OR070, P151, P153, P155, P223

Letourneur, D, OR066

Lettner, T, P058

Leu, C H, P240

Leuchs, B, OR009

Leumann, C, P183

Levälampi, O, P027

Levijoki, J, OR058

Li, L, OR045

Lichter, P, P021

Liebhaber, S, P200

Liefhebber, J M, P060

Lieubeau, B, OR011

Ligat, L, OR002

Liikanen, I, P222

Liimatainen, T, OR067, P030

Liivrand, M, P042

Lillegard, J B, OR046

Lin, C Y, OR059

Lin, G, P124

Linden, R M, P237

Link, B, P239

Lipponen, E, P151

Lipponen, E M, OR070, P153

Liran, A, OR036

Liu, F, P158

Liu, J, OR041

Liu, P Q, OR045

Liu, T, P004, P006

Loader, J, P188

Locafaro, G, P086

Locatelli, F, P086

Lodder, E M, P040

Logan, G J, OR075, P098, P099

Logg, C R, P268

Logtenstein, R, P060

Lomas-Romero, I, P208

Lombardo, A, OR012, OR019, OR042, P005, P054, P227

Lopes, C D F, P166

López, J A, OR071

Lópe-Andújar, R, P169

Lopez-Franco, E, OR005
Lopez-Gordo, E, P234

Lopez-Manzaneda, S, P001, P007

Lopez, F, OR002

Lopez, V M, P023

Lorant, J, OR011

Loskog, A, P089, P106

Lostal, W, P160

Lottonen-Raikaslehto, L, P046

Louboutin, J-P, P124

Loucari, C, P003

Löw, R, P258

Lowe, R M, P104

Lozano, M L, OR034, P055

Lu, B, OR074, P123

Luce, E, P207

Luciani, M, OR032

Ludewig, S, OR033

Luhmann, U F, OR021

Lulka, H, OR002

Lumen, D, P018

Lundin, K, P056

Lundin, K E, P068, P170

Lunstad, B D, OR030

Luostarinen, A, P101

Lupo-Stanghellini, M T, OR065, P095

Lüttge, D, P200

Luyckx, E, P219

Määttä, A, P276

Mähönen, M, P155

Mäkinen, K, OR067

Mäkinen, P, P036, P101, P229

Mañu-Pereira, M M, P007

Mack, D L, P158

Madeja, Z, P074, P215

Maepa, M B, P256

Maetzig, T, P226

Magarkar, A, OR052, P088, P103

Maggio, I, OR041

Maggioni, L, P128

Magis, W, P069

Magot, A, OR011

Mahalatchimy, A, OR061

Maina, V, P092

Mak, K Y, P265

Makino, A, OR074, P255

Makkonen, K E, OR070

Malam, Z, OR040

Malech, H L, OR045

Maliandi, M V, P221

Malinen, J, OR070, P155

Mallol, C, P131, P134

Malnero, C M, P023

Mamchaoui, K, OR040, OR041

Mandelli, G, P242

Manfredini, M, P028

Mangia, P, P028, P029

Manolaras, I, OR039

Manta, S, OR051

Mao, S A, OR046

Maouche-Chrétien, L, P207

Marcó, S, P128

Marchenko, Y, P102

Margulis, B, P102 
Marie, C, P066, P177, P182, P184

Marin, V, OR012, P144

Markmann, S, P127

Martín, F, P230

Martier, R, P060

Martikainen, A, OR070, P155

Martikainen, M, P270

Martin-Lopez, M, P208

Martin, D, P069

Martin, F, P082

Martin, J, P275

Martin, S, OR043, P111, P158

Martino, S, OR032, P129

Masat, E, P118

Mastaglio, S, OR065

Mato-Berciano, A, P221

Matsui, A, P173

Matsumoto, Y, P255

Mattila, M, P045

Maunder, H E, OR078

Mautner, V, P016

Mavilio, F, OR069, OR076, P061, P111, P146, P148, P158

Mayr, E, P073

Mazarakis, N D, OR039

Mazda, O, P079

McGovern, M, OR049

McGuffog, C, P099

McMenamin, D, P253

Mehta, V, P275

Meier, C, P078

Mencia, A, P070

Mendel, M, P046

Mendes-Madeira, A, P181

Mendoza-Londono4, R, OR040

Meneghini, V, OR032

Meng, H, P158

Mentzen, W, OR032

Merentie, M, P046

Merisalo-Soikkeli, M, P018

Merlin, S, P005

Merten, O W, P146, P149, P252

Mervaala, E, OR058

Mesa-Nuñez, C, OR050

Messina, G, OR042

Messina, M, P005

Meunier, S, OR020

Miari, R, OR036, P115

Midoux, P, OR051, P180

Migliavacca, M, P081

Mignet, N, OR051

Miguel, A, P034, P169

Mikkelsen, J G, P186, P257

Milani, M, P006, P227

Milenova, I, P089, P106

Miletic, H, P013

Miller, J, P189

Mineno, J, P011, P091

Mingozzi, F, OR069, P118, P148, P158

Miniarikova, J, OR029

Miskey, C, OR050

Mitra, K., P274

Mitrophanous, K, P188

Mitrophanous, K A, OR078
Miyahara, Y, P091

Mizukami, H, P010

Moal, C, OR043

Moawadh, M S, OR075, P098

Moccetti, T, OR060

Mock, U, OR072

Moestrup, S K, P044

Molas, M, P128

Mole, S E, OR021

Moncaubeig, F, P138

Montalvá, E, P169

Montepeloso, A, P129

Montiel, M A, P208

Montini, E, OR023, P125, P262

Montini, E., P274

Monyer, H, OR073

Morais, J, P167

Morandi, P, P028

Moranvillier, I, P217

Moreau-Gaudry, F, P217

Morena, F, OR032, P129

Moreno, P M D, P166

Moreno, R, P022, P089, P106

Morgan, M, OR024

Morinaga, T, P014

Moritz, T, OR055, P200, P260

Mormin, M, P114, P149

Morró, M, P134

Morris, K, OR028

Morrison, C J, P154

Moser, M, P219

Moss, D, P266

Most, P, OR009

Mostoslavsky, G, P205

Mota, J PB, P150

Motas, S, P128

Moullier, P, P141, P181

Mouly, V, OR040, OR042

Mousiadou, A, P198

Mucci, A, OR055

Muik, A, OR054

Muller, A, P151

Müller, A, OR070

Müller, F J, P226

Müller, O J, OR009

Müller, U C, OR033

Münch, R, OR073

Muñoz, D, P069

Muñoz, M P, P209

Muñoz, S, P131

Munoz-Gomez, M, P123

Muntoni, F, OR040

Muraro, M G, OR064

Murauer, E M, P071, P072, P073

Muravjev, A N, P199, P203

Muriel-Ramos, M, P007

Murillas, R, P070

Murray, G, OR049

Muss, W, P058

Mussalo, H, OR067

Mussolino, C, OR014, P003, P053, P074

Mustjoki, S, P101

Mykkänen, J, P223, P245
Närvänen, O, P151

Nabholz, N, P085

Naeem, A, P187

Nakazawa, Y, P094

Naldini, L, OR003, OR012, OR019, OR042, OR048, P004, P005, P006, P054, P081, P086, P090, P125, P129, P132, P227, P242, P261

Nambiar, B, P008

Nashchekina, J A, P199

Nashchekina, Y A, P195, P203

Nathwani, A C, P135

Nauwynck, L, P146

Navarro, S, OR034, P007, P053

Nedellec, S, P181

Neil, G A, OR036, P115

Nestola, P, P150

Netesov, S V, P254

Nettelbeck, D, P112

Newrzela, S, OR054

$\mathrm{Ni}, \mathrm{M}, \mathrm{P} 249$

Nichols, T, P006

Nicklin, S A, P234

Nickolay, L E, OR072

Nieminen, T, OR063, P038, P049

Niittykoski, M, P270

Nikolaev, B, P102

Nikonov, P O, P195

Niskanen, H, P038, P042, P043, P052

Noé, V, P034

Norelli, M, OR003, P090

Nork, T M, P189

Norol, F, OR066

Notarangelo, L D, OR035

Notaridou, M, P135

Nousiainen, A, P229

Noviello, M, P086

Nowrouzi, A, P057

Nozza, S, OR023

Nurminen, E M, OR070, P151, P153

Nusco, E, P133

Nyberg, S L, OR046

Nygårdas, M, P105

O'Callaghan, M, P158

O'Connor, J E, P210

O'Donnell, P, P124

O'Leary, C, P122

O'Riordan, C, P008

Ohmine, S, P196

Ohmori, T, P010

Okamoto, S, P011, P014, P091

Oksanen, M, P222

Olgasi, C, P005

Olgun, H B, P137

Oliveira, A V, P167, P175

Oliveira, G, OR065, P095

Olsson, V, P193

Onodera, M, P094

Oosterwerff, E, P040

Orito, Y, P091

Orlova, N V, P199, P203

Orman, I, P002

Ortega, L Cócera, P040 
Osborn, M J, P093

Oshimura, M, OR042

Otonkoski, T, OR015, P206

Otsu, M, P094

Otte, K, OR007

Oviedo, V M, P232

Ozawa, K, P010

Ozcan, A G, P076, P077, P204

Paavilainen, H, P105, P233

Pabon, Y V, P068

Pacouret, S, OR077

Paíno, C L, P209

Palacios, I, P039

Paldi, A, OR027

Palfi, S, P188

Panchal, N, P117

Pantelidou, C, OR053

Pantoglou, J, P121

Parenti, G C, OR031

Parezanovic, V, P242

Parini, A, P048

Parker, A L, P224

Parker, H, P122

Parker, N, P151

Parker, N R, OR070, P153

Parlar, A, OR013

Parola, C, OR016

Parviainen, S, P017, P112, P236

Parviainen, V, P046

Paschon, D E, OR045

Passerini, L, OR023, OR048

Pastore, L, P047

Patarroyo-White, S, P004

Patel, P, P016

Patsali, P, P003

Paul, M, OR075, P098, P099

Paulis, M, OR032

Paulo, S, P223, P245

Pavan, W J, P126

Paw, M, P074

Pearl, J R, OR045

Pearson, R A, OR021, P187

Peccatori, J, OR065, P095

Peces-Barba, M, P210

Peebles, D M, P275

Pêgo, A P, P166

Peiffer, S, P028

Peixoto, C, P150, P156

Peking, P, P058, P073

Pellin, D, P269

Penaud-Budloo, M, P141

Peng, J S, OR027

Peng, K W, OR054

Péréon, Y, OR011

Pequignot, M O, P080, P085

Peral, F, P142

Perdigão, P, P063

Pereira, A C, P205

Pereira, S, OR040

Peruta, M Della, P135

Peshwa, M V, OR045

Pesonen, S, P027

Pesonen, S K, P222
Petri, K, P093, P237

Petry, H, OR005, OR029, P060

Peyret, A, OR051

Pichard, V, P181

Pichon, C, OR051, P180

Pichon, F, P061

Piersanti, S, P191

Pigeon, L, P180

Pike-Overzet, K, P108

Pinkert, S, P271, P272

Pino-Barrio, M J, P053

Pinto, C, P191

Piouka, N, P211

Piovan, C, P144

Piraino, S, P008

Piras, F, P261

Pitkin, M, P197

Podhajcer, O L, OR004, P023

Pogson, M, OR016

Poinsignon, C, OR027, P024, P114

Poirier, B, OR031

Poirot, L, P002, P055

Poletti, V, P061, P111

Poli, G, OR023

Poliani, P L, P092

Poljanskaya, G G, P216

Pollen, M, P151

Ponsaerts, P, OR068, P219

Porfiri, E, P016

Porkka, K, P101

Porteous, D J, OR049

Porter, F D, P126

Porteus, M, OR048

Porteus, M H, OR030

Potokin, I, P197

Pötschke, F J, P058

Poulard, K, P158

Poupiot, J, P159, P162, P164

Povlich, S F, P154

Pozzuto, T, P272

Préat, V, P097

Prakash, V, P121

Prat-Souteyrand, C, P182

Prat, M, P005

Prats, A C, P048

Precigout, G, P251

Prieto, J, OR005

Provost, N, P181

Pryadkina, M, P160

Pshezhetsky, A, P122

Pujol, F, P048

Pule, M, OR072

Pulkkinen, H, P038

Purcell, A, P098

Pützer, B M, P078

Qasim, W, OR072

$\mathrm{Qu}, \mathrm{J}, \mathrm{P} 012$

Quintana-Bustamante, O, P002, P055

Quiviger, M, P177

Räsänen, E, OR070, P153

Raake, P W, OR009

Radlwimmer, B, P021
Radrizzani, M, OR060

Ragazzi, M, OR042

Rajapaksha, I G, P265

Ralph, S, P188

Ramalho, A S, P264

Ramirez, J C, P001

Ramirez, M, P022

Ranki, T, P027

Ranucci, S, P081

Rath, T, P057

Rautsi, O, P151

Ravassard, P, P246

Raya, A, OR014, P005

Rebar, E, OR017

Rebar, E J, OR045

Recchia, A, P061, P129

Reddy, K B, OR078

Reddy, S, OR016

Reekmans, K, OR068, P219

Reetz, J, P078

Reginald, A, OR001, P198, P211

Reginato, S, OR064

Reichenbach, J, P116

Reik, A, OR017, OR045

Renaud-Gabardos, E, P048

Rengstl, B, OR054

Reniers, A, P138

Restelli, S, OR048

Revaud, D, P109

Riba, M, P261

Ribera, A, P128, P131

Ricci, L Basso, OR056, P269

Ricciardelli, I, OR037

Richard, I, P159, P160, P161, P162, P164

Richards, J, P104

Richaud-Patin, Y, OR014

Riecken, K, P013

Rigau, V, P085

Rinaldo, P, OR046

Rincon, E, P022

Rincon, M, OR038

Rio, M Del, P070

Rio, P, OR034, P053, P054

Ripoll, C, P246

Ritamo, I, OR058

Rivière, J, OR037

Riviere, I, P012

Rizzardi, G P, P028, P029, P144

Robert, L, P080, P085

Roberts, A, P121

Roberts, T C, OR028, P035

Robriquet, F, P157

Roca, C, P128

Rocca, C J, P121

Rodo, J, P131

Rodríguez-Borlado, L, P039

Rodrigues, A F, P145, P244, P247

Rodrigues, A. F., P273

Rodriguez-Fornes, F, P002, P055

Rodriguez-Milla, M A, P022

Rodriguez, M A Pañeda, OR005

Roederer, J P, OR033

Röger, C, P272 
Rolling, F, P181

Roman-Rodriguez, F J, P054

Romaniuk, D, OR052, P088

Roncalli, J, P048

Roobrouck, V, P219

Rosada, C, P257

Rosado, R, P039

Rossetti, F, P029

Rossi, A, P081

Rossi, C, P242

Rossi, C A, P275

Rossi, P, P081

Rossi, S, P250

Rothe, M, P111

Rothmann, T, P186

Rotondaro, C, OR004

Roudaut, C, P159, P160

Rouger, K, OR011, P157

Rouillon, J, P164

Roujeau, T, OR031

Rousseau, B, P217

Roy, S, OR030

Röyttä, M, P105

Ruane, T, P124

Rubanyi, G M, OR010

Ruberte, J, P128

Rudilosso, L, OR023, P262

Rudolph, C, OR050

Ruggiero, E, OR065, P093

Russell, S J, OR046

Ryan, D E, OR030

Sánchez-Domínguez, R, P210

Sánchez-Hernández, S, P230

Sánchez, B, P039

Saari, H, P019

Saarimäki-Vire, J, OR015, P206

Sabater, L, P169

Sabbah-Petrover, E, OR025

Sacchetti, L, P047

Sacchi, V, OR064

Sacristan, V, P131, P134

Sadelain, M, P012

Saga, Y, P010

Saggio, I, P191

Sah, S K, P107, P267

Saint-Laurent, N, OR002

Sakata, Y, P010

Saksela, K, P270

Sakuma, T, OR006

Saland, E, OR002

Sallinen, H, P030

Salonen, T, OR070, P155

Salvatierra, E, OR004

Salvatore, F, P047

Samara-Kuko, E, OR038

Sanchez-Dominguez, R, OR034, P001, P002, P007, P053

Sanchez-Hernandez, S, P082

Sankkila, L, OR058

Sanlioglu, S, P137, P228, P241

Santa-Marta, M, P063

Santermans, E, OR068

Santilli, G, P116
Sanvito, F, OR032, P132, P242

Sarcar, S, OR038

Sardon, D, P022

Sarry, E, OR025, P087, P109

Sarry, J E, OR002

Sarvela, T, OR052, P088, P103

Saudemont, A, OR003, P090

Sauer, A, P242

Sauer, A V, P104

Saunders, C J, OR049

Savola, P, P222

Savontaus, M, P045

Savvatis, K, P271

Savy, A, P146, P252

Sayitoglu, E C, OR013

Scala, S, OR056, P269

Scaramuzza, S, OR056, P081, P242

Scavullo, C, P144

Schambach, A, OR024, OR055, P074, P111, P226, P260

Scheiflinger, F, P227

Schenkwein, D, P229, P239

Scherman, D, P066, P177, P182, P184

Scheule, R K, OR049

Schiavo, G, P191

Schinco, P, P005

Schinkel, S, OR009

Schiroli, G, OR012, OR019, P054

Schleder, C, OR011

Schleef, M, OR050, P139, P152

Schmeer, M, OR050, P152

Schmidt, A, P078

Schmidt, M, OR005, OR022, OR065, P057, P083, P093, P121, P237, P238, P239

Schmidtke, M, P271

Schmolka, N., P273

Schneider, I, OR054

Schnoedt, M, P152

Scholl, C, P057

Schott, J W, P226, P260

Schreiber, C A, OR006

Schwartz, D, OR036

Scripps, V, OR078

Searle, P F, P016

Seeger, M, OR073

Segovia, J C, OR034, P001, P002, P007, P055, P210

Segura, B, P130, P232

Sekine, I, P014

Sekula, M, P074

Selamet, Y, P064

Selvamani, R, P139, P152

Semenova, N Y, P199

Sendra, L, P034, P169

Seng, S H, P008

Senturk, G H, OR013

Sereni, L, OR035

Sergi, L Sergi, P081

Sergijenko, A, OR044

Sermet-Gaudelus, I, P264

Serra, M, OR071, P156

Servais, L, P164
Sevilla, J, OR034, P007

Sganga, L, OR004

Shapir, N, OR036, P115

Sharafi, P, P212, P214

Sharland, A F, OR075, P098, P099, P265

Shatil, T, P115

Shaw, R, OR070, P151, P153, P223, P245

Shejhov, M G, P199

Shevtsov, M, P102, P197

Shevtsov, M A, P199, P203

Shiau, A L, P119, P240

Shiku, H, P011, P091

Shimada, H, P014

Shingyoji, M, P014

Shubina, A N, P176

Sicard, P, P048

Siclari, F, OR060

Sikora, T, P124

Siler, U, P116

Siljander, P, OR058, P019

Silva-Santos, B., P273

Silva, G A, P167, P174, P175, P190

Silva, R, P150, P156

Simão, D, P191

Simão, S, P190

Simmonds, N J, OR049

Simonelli, F, P250

Singh, M, P165

Sit, K, P074, P215

Sitia, G, OR019

Siurala, M, P017, P018, P112, P236

Skokowa, J, P200

Slita, A A, P065

Smith, A J, OR021

Smith, C I E, P056

Smith, C I Edvard, P068

Smith, C IE, P170

Smith, S, OR017

Snoek, S A, OR057

Snyder, J M, P158

Sobczyk, A, P185, P246

Sobrevals, L, P221

Sodeik, B, P105

Soderini, A, P023

Soheili, T, OR037

Somanathan, S, P253

Somella, A, P250

Soncin, S, OR060

Soncin, W, P029

Song, A, OR045, P026

Sookdeo, C, P008

Sousa, M FQ, OR071

Southwell, A, OR029

Spinozzi, G, P125, P262

Spoerl, A, OR029

Spronck, L, OR005

Staal, F J, P108

Stahlhut, M, OR024

Stamatoyannopoulos, G., P274

Staton, H, P104

Stavropoulos, J, OR040

Stavrou, E F., P177 
Steel, M, P147

Stefanski, J, P012

Stefanucci, L, OR041

Steichen, C, P205, P207

Steinfeld, I, OR030

Steinhoff, G, P168

Stenderup, K, P257

Stenler, S, P170

Stephan, C, P239

Stephanou, C, P003

Stewart, H, P188

Stiefel, S, OR007

Stockholm, D, OR027

Stradella, E, P242

Strande, J L, P158

Stupka, E, P261

Sullivan, T, OR017

Sultan, S, OR001, P198, P211

Sumner-Jones, S G, OR049

Surralles, J, OR034

Sutlu, T, OR013

Suutari, T, OR052, P088

Suzuki, T, OR055

Svensson, E, P089, P106

Svinartchouk, F, P164, P231

Sweeney, C, OR045

Tähtinen, S, P017, P018, P222

Taavitsainen, J, OR008

Tada, Y, P014

Tagawa, M, P014

Tak, P P, OR057, P220

Takagi-Kimura, M, P268

Takesako, K, P091

Talmon, M, P005

Tambussi, G, OR023

Tan, H L, P040

Tan, S, OR017, P004

Tarantal, A F, P124

Tarasova, M V, P254

Tardieu, M, OR031

Tarvainen, S, OR008, OR067

Tasyurek, H M, P137, P228, P241

Tatoris, S, OR078

Tatsumi, K, P014

Tavi, P, P050

Tay, S S, OR075, P098, P099

Tayton-Martin, H, P266

Tedesco, F S, OR042

Telaar, M, P139

Tenderini, E, P125

Tenderini, E., P274

Terzic, A, P196

Theobald, N, OR045

Thomasson, N, OR020

Thrasher, A, OR072, P111, P116

Thrasher, A J, OR035, P062, P121

Thumann, G, P066, P182, P184

Tian, S, P184

Tibaldi, L, P087

Tiboni, F, P242

Tipanee, J, OR038

Tirronen, A, P038

Tirronen, A K, P050
Titeux, M, P258

Tobalem, S, P182

Todorov, G, P249

Toivanen, P I, OR063, P038, P049

Tolar, J, P093

Tolosa, E, OR014

Tomás, H A, P145, P247

Tomczyk, M, P113

Tomonaga, K, OR074, P255

Tonlorenzi, R, OR042

Tonne, J M, P123, P196

Toriello, E, P250

Torre, T, OR060

Torres, R, P001

Torres, V, OR047

Torresi, J, P265

Toscano, M G, P082

Toussaint, H, OR025

Towler, J, P104

Trani, M, OR064

Trapani, I, P250

Trapnell, B, OR055

Traversari, C, OR065, P028, P029

Trono, D, P081

Tsalenko, A, OR030

Tshilenge, K T, P181

Tsukahara, T, P010

Tuimala, J, OR058

Tuppurainen, L, P030

Turchetto, L, OR060

Turunen, H T, OR077, P235

Turunen, M P, OR018, OR028, P035, P036

Tuunanen, T, OR070

Uchibori, R, P010

Uchida, S, OR059, P173

Uchiyama, T, P094

Uckan-Cetinkaya, D, P212, P213, $\mathrm{P} 214$

Uckan, D, P204

Uckert, W, P100

Ungari, S, P129

Uno, N, OR042

Urabe, M, P010

Uretsky, S, OR020

Urnov, F D, OR017, OR045

Urtti, A, OR052, P088, P103

Usadel, S, P239

Ustinov, J, P206

Uusi-Kerttula, H K, P224

Väänänen, M, OR028, P035, P036

Vago, L, OR065, P095

Vähä-Koskela, M, P015, P017, P018, P270

Vainchenker, W, OR066

Valencia, R, P116

Valente, M M, OR048

Valeri, F, P005

Valkama, A, OR070

Valkama, A J, P155

Valkama, T, P041

Vallanti, G, P028, P029
Valle, P Della, P006

Valmu, L, OR058

Valonen, P, OR070, P153

van Baarsen, L, OR057

Van Camp, N, P188

van Dongen, J J, P108

van Geldorp, M, OR057

van Geldorp, M A, P220

van Ittersum, J, OR057

van Til, N P, P092

Van de Loo, F AJ, P110

Van den Berg, W B, P110

Van den Haute, C, P264

van der Burg, M, P108

Van der Linden, A, OR068, P219

VandenDriessche, T, OR038, P006

Vandenberghe, L H, OR077, P235

Vandermeulen, G, P097

Vanvarenberg, K, P097

Varela-Moreira, A, P166

Vassalli, G, OR060

Vassilev, L, P027

Vattulainen, M, P038

Vazquez, F, P022

Vázquez, J, OR071

Végh, A MD, P040

Velazquez, D, P130

Vellosillo, L, P209

Venditti, C P, P126

Vendomèle, J, P114

Vepsäläinen, S, P151

Verbeeck, L, P219

Verfaillie, C, P219

Verhoeyen, E, OR037

Verhoye, M, OR068

Verkerk, A O, P040

Vermeij, E A, P110

Veron, P, P158

Verras, M, P177

Vervoordeldonk, M J, OR057, P220

Veselova, T V, P195

Vezzoli, M, P132, P242

Vicenzi, E, OR023

Vidovic, D, P264

Vieira, C R, OR078

Vignaud, A, OR043, P111

Vignolle-Vidoni, A, OR002

Viitala, T, OR052, P019, P088

Vijay, S, P147, P189, P249

Vilà, L, P134

Villa, A, OR019, OR032, OR035, P092

Villa, C, P242

Villain, L, P150

Vincent, K, P008

Viney, R, P016

Vinogradova, T I, P199, P203

Visigalli, I, P132, P242

Vitale, A, OR052, P015, P088, P225

Vitovskaja, M L, P199

Vives-Corrons, J L, P007

Voit, T, OR040, P164

Volpin, M., P274

Volz, C, OR009 
Von Kalle, C, OR065, P083

von Kalle, C, OR005, OR022, P057,

P093, P121, P237, P238, P239

von und zu Fraunberg, M, P270

Voronina, N, P168

Vranckx, L, P263

Vuolanto, A, P027

Vuorimaa-Laukkanen, E, P019

Vuorio, T, P052

Wadsworth, S C, P154

Wagemaker, G, P092, P212

Waindok, A, OR016

Waller, M D, OR049

Wally, V, P058

Walter, P, P066

Walther, W, OR050

Wang, C, OR075, P098, P099

Wang, C R, OR027

Wang, J, P121

Wang, L, P253

Wang, P, P124

Wang, Q, P253

Wang, W, P021

Wang, X, P012

Wang, Z, P099

Wartiovaara, K, OR015, P069

Wasielewska, T, P012

Watanabe, N, P094

Weber, A, P207

Weber, C, P239

Weber, H L, OR004

Weber, M, P181

Weinberg, M, OR028

Wells, D J, P170

Welstead, G G, P075

Wengel, J, P056, P068
Wenthe, J, P089

Werbajh, S, OR004

West, E L, P187

Westaby, S, OR001

Weyer, S W, OR033

Wiekmeijer, A S, P108

Wilkening, S, OR005, OR022, P083, P237

Wilkens, A B, OR030

Wilkinson, F, OR044, P122

Williams, D, P266

Williams, I M, P126

Wilmes, G M, P154

Wilson, J M, P124, P253

Wirth, G, P037, P044, P276

Wisher, M H, P140

Witt, M, P078

Wojciechowski, S, P229

Wojtal, D, OR040

Wolvers-Tettero, I L, P108

Wong, B, P164

Wong, T W.Y., OR040

Wood, M JA, P170

Woodburn, K, P147, P249

Woodburn, K W, P189

Wree, A, P078

Wright, J, OR078

Wrona, D, P116

$\mathrm{Wu}, \mathrm{C}$ L, OR027, P240

Wynn, R, OR044

Yakovleva, L, P102

Yakovleva, T K, P216

Yamamoto, Y, P255

Yang, L, P158

Yang, M L, P240

Yáñez-Muñoz, R J, P121

Yañez, R, OR034, P210
Yao, R, OR027

Yasukawa, M, P011, P091

Ylä-Herttuala, S, OR028, OR063, OR067, OR070, P030, P035, P036, P038, P041, P042, P043, P044, P046, P049, P050, P052, P101, P151, P153, P155, P193, P223, P229, P276

Ylösmäki, E, P270

Yla-Herttuala, S, OR008, P037

Yliperttula, M, OR052, P019, P088

Yoneyama, M, P091

$\mathrm{Yu}, \mathrm{H}, \mathrm{P} 124, \mathrm{P} 253$

Yudintceva, N, P197

Yudintceva, N M, P203

Yun, C O, P267

Zabolotnich, N V, P199

Zachary, I, P275

Zafar, S, P236

Zafeirou, D, OR031

Zafir-Lavie, I, P115

Zaghloul, E, P056

Zain, R, P056, P068

Zanella, I, OR029

Zarember, K A, OR045

Zemmar, A, P143

Zerah, M, OR031

Zhang, F, P111

Zhou, Q, P004

Zhu, Y, P124

Zimmer, V, OR029

Zinn, E, OR077, P235

Zocevic, A, P164

Zolla, W C, P154

Zonari, E, P081

Zuba-Surma, E K, P074, P215

Zuber, C, OR011 